\title{
Research Article \\ Dislocation Nucleation and Pileup under a Wedge Contact at Nanoscale
}

\author{
Y. F. Gao' ${ }^{1}$ and J. Lou ${ }^{2}$ \\ ${ }^{1}$ Department of Materials Science and Engineering, University of Tennessee, Knoxville, TN 37996, USA \\ ${ }^{2}$ Department of Mechanical Engineering and Materials Science, Rice University, Houston, TX 77251, USA
}

Correspondence should be addressed to Y. F. Gao, ygao7@utk.edu

Received 5 November 2007; Accepted 18 January 2008

Recommended by Junlan Wang

Indentation responses of crystalline materials have been found to be radically different at micrometer and nanometer scales. The latter is usually thought to be controlled by the nucleation of dislocations. To explore this physical process, a dislocation mechanics study is performed to determine the conditions for the nucleation of a finite number of dislocations under a two-dimensional wedge indenter, using the Rice-Thomson nucleation criterion. The configurational force on the dislocation consists of the applied force, the image force, and the interaction force between dislocations. Dislocations reach equilibrium positions when the total driving force equals the effective Peierls stress, giving a set of nonlinear equations that can be solved using the Newton-Raphson method. When the apex angle of the wedge indenter increases, the critical contact size for dislocation nucleation increases rapidly, indicating that dislocation multiplication near a blunt wedge tip is extremely difficult. This geometric dependence agrees well with experimental findings.

Copyright (C) 2008 Y. F. Gao and J. Lou. This is an open access article distributed under the Creative Commons Attribution License, which permits unrestricted use, distribution, and reproduction in any medium, provided the original work is properly cited.

\section{INTRODUCTION}

The ability to quantitatively model mesoscopic material behavior is a critical step in understanding reliability analysis and material design for applications ranging from aerospace components to nanoelectronics. Many experimental studies in the past decade have shown that the contact plasticity is size-dependent (i.e., indentation size effects, ISEs) and thus is essentially a multiscale problem [1-7]. This phenomenon is believed to be due to the collective behavior of dislocation nucleation and storage under the contact. Statistical theories have been developed to understand the dislocation microstructures that form and evolve during contact events, but most of these theories are only applicable when the contact size is larger than micrometers. A number of recent experiments demonstrate the importance of individual dislocation nucleation events, such as pop-in excursions on the loaddisplacement curves $[7,8]$, which are thought to govern the indentation size effects at nanoscale, while the size effects at microscale are associated with strain gradient effects and geometrically necessary dislocations $[1,2,7]$.

A variety of analyses have been carried out using discrete dislocation and phenomenological strain gradient plasticity to model ISE across the length scales $[9,10]$. At larger length scale (submicron to micrometer scales), both strain gradient plasticity theories that are based on geometrically necessary dislocations and discrete dislocation simulations have provided important insights in understanding the dependence of microhardness on contact size. Many recent theories aim to modify these theories for nanohardness measurements $[4,10]$, but the connection to the dislocation microstructure still remains elusive. At sufficiently small-length scale, the size of the contact region under high stress may be comparable to the dislocation spacing, so that the indentation behavior may enter the dislocation-nucleation-controlled regime. This work attempts to explore this line from the study of the dislocation nucleation and pileup under a two-dimensional wedge contact tip.

\section{PROBLEM FORMULATION AND SOLUTION METHOD}

Figure 1 gives the problem definition where the apex half angle is $\alpha$, the applied load is $P$ (per unit length out of the plane), and the contact size is $a$. Suppose that a dislocation 


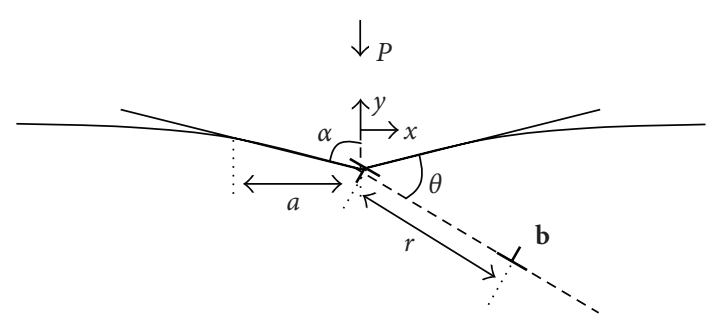

FIGURE 1: Schematic illustration and geometric conventions of the contact problem.

$\mathbf{b}=b(\cos \theta, \sin \theta, 0)^{\mathrm{T}}$ can be nucleated from the indenter tip, then a negative dislocation $-\mathbf{b}$ will be left. This dislocation pair will lead to tilting of the indenter, but such a modification is usually very small [11] and will be neglected in this work. The micromechanical model consists of three parts: (1) the determination of the configurational force on the dislocation, (2) the use of the Rice-Thomson criterion for dislocation nucleation, and (3) the calculation of the equilibrium dislocation positions. In the continuum dislocation modeling, we use the Rice-Thomson dislocation nucleation criterion $[12,13]$. If the driving force on a dislocation at a distance $\eta$ from a stress concentration is greater than the Peierls force, the dislocation can be emitted from the step. The dislocation glides only if the driving force overcomes the Peierls force. In the Rice-Thomson model, parameter $\eta$ characterizes the size of the dislocation emission process zone. We can either regard it as a material parameter or determine it by atomistic simulations. With the information of $\eta$ and the effective Peierls stress $\tau_{p}$, we can easily decide whether a dislocation nucleates and where the equilibrium position of the nucleated dislocation would be, as long as the driving force on the dislocation is determined.

The driving force on the dislocation $\mathbf{b}$ has three parts:

$$
J=J_{\text {appl }}+J_{\text {image }}+J_{\text {int }},
$$

where $J_{\text {appl }}$ is the applied driving force, $J_{\text {image }}$ is the image force, and $J_{\text {int }}$ is the interaction force due to the dislocation - $\mathbf{b}$ and/or other pairs of dislocations. The applied driving force can be computed from the stress field [11]:

$$
\begin{aligned}
& \sigma_{x x}=\frac{2 y}{\pi a}\left(\frac{\mu}{1-\nu}\right) \frac{\cot \alpha}{\pi} \int_{-1}^{1} \frac{\cosh ^{-1}(1 / t)(x / a-t)^{2} d t}{\left[(x / a-t)^{2}+(y / a)^{2}\right]^{2}}, \\
& \sigma_{y y}=\frac{2 y^{3}}{\pi a^{3}}\left(\frac{\mu}{1-\nu}\right) \frac{\cot \alpha}{\pi} \int_{-1}^{1} \frac{\cosh ^{-1}(1 / t) d t}{\left[(x / a-t)^{2}+(y / a)^{2}\right]^{2}}, \\
& \sigma_{x y}=\frac{2 y^{2}}{\pi a^{2}}\left(\frac{\mu}{1-\nu}\right) \frac{\cot \alpha}{\pi} \int_{-1}^{1} \frac{\cosh ^{-1}(1 / t)(x / a-t) d t}{\left[(x / a-t)^{2}+(y / a)^{2}\right]^{2}},
\end{aligned}
$$

and the Peach-Koehler formula:

$$
J_{\text {appl }}=\left[(\boldsymbol{\sigma} \cdot \mathbf{b}) \times(0,0,1)^{\mathrm{T}}\right] \cdot(\cos \theta, \sin \theta, 0)^{\mathrm{T}} .
$$

Those curves of $J_{\text {appl }}(1-\nu) / \mu b$ against $r / b$ will collapse onto each other by replotting $J_{\text {appl }}(1-\nu) / \mu b$ against $r / a$, clearly because the indenter is self-similar. The state of stress at the apex comprises a finite shear stress superposed on an infinite hydrostatic pressure. The applied driving force at the apex can be written as

$$
\left.\frac{(1-v)}{\mu b} J_{\text {appl }}\right|_{r \rightarrow 0}=\frac{\cot \alpha}{\pi} f(\theta),
$$

where $f(\theta)$ is a dimensionless function that only depends on the slip-plane angle of the order of unity. This stress state is radically different from the stress singularity caused by a crack tip, or a flat-ended punch under normal contact, or an arbitrary indenter under tangential contact $[14,15]$. The implications on the dislocation nucleation behavior will be discussed shortly.

The image force can be determined by considering two opposing semi-infinite cracks in an undislocated plane, subjected to tractions that will cancel the tractions induced by the dislocation in an uncracked plane. Using the complex function analysis, the stress field at $z=x+i y$, caused by a dislocation $\mathbf{b}$ at $z_{0}=x_{0}+i y_{0}$, in an uncracked plane is given by [16]

$$
\begin{aligned}
\tilde{\sigma}_{x x}+\tilde{\sigma}_{y y} & =2[\Phi(z)+\overline{\Phi(z)}], \\
\tilde{\sigma}_{y y}+i \tilde{\sigma}_{x y} & =\overline{\Phi(z)}+\Omega(z)+(\bar{z}-z) \Phi^{\prime}(z),
\end{aligned}
$$

where the holomorphic complex functions $\Phi$ and $\Omega$ are

$$
\begin{aligned}
& \Phi(z)=\frac{B}{z-z_{0}}, \quad \Omega(z)=B \frac{\bar{z}_{0}-z_{0}}{\left(z-z_{0}\right)^{2}}+\frac{\bar{B}}{z-z_{0}}, \\
& B=\frac{\mu}{\pi i(1+\kappa)}\left(b_{x}+i b_{y}\right)=\left(\frac{\mu}{1-\nu}\right) \frac{1}{4 \pi}\left(-i b_{x}+b_{y}\right),
\end{aligned}
$$

and $\kappa=3-4 \nu$ for plane strain.

Now consider an undislocated plane with two opposing semi-infinite cracks, subjected to the corrected tractions $-\widetilde{\boldsymbol{\sigma}}(|x|>a, y=0)$. Following Tada's solution 4.5 [17], the correction stress field can be determined by

$$
\begin{aligned}
& Z_{\mathrm{I}}(z)=\left(\int_{-\infty}^{-a}+\int_{a}^{\infty}\right) d t \frac{-\tilde{\sigma}_{y y}(t, 0)}{\pi} \frac{\sqrt{t^{2}-a^{2}}}{\sqrt{a^{2}-z^{2}}}\left(\frac{1}{t-z}+\frac{2 z}{a^{2}}\right), \\
& Z_{\mathrm{II}}(z)=\left(\int_{-\infty}^{-a}+\int_{a}^{\infty}\right) d t \frac{\tilde{\sigma}_{x y}(t, 0)}{\pi} \frac{\sqrt{t^{2}-a^{2}}}{\sqrt{a^{2}-z^{2}}}\left(\frac{1}{t-z}\right),
\end{aligned}
$$

where $Z_{\mathrm{I}}(z)$ and $Z_{\mathrm{II}}(z)$ are the mode-I and mode-II Westergaard stress functions, respectively. The stress components inside the substrate are

$$
\begin{aligned}
& \hat{\sigma}_{x x}=\left(\operatorname{Re} Z_{\mathrm{I}}-y \operatorname{Im} Z_{\mathrm{I}}^{\prime}\right)+\left(2 \operatorname{Im} Z_{\mathrm{II}}+y \operatorname{Re} Z_{\mathrm{II}}^{\prime}\right), \\
& \hat{\sigma}_{y y}=\left(\operatorname{Re} Z_{\mathrm{I}}+y \operatorname{Im} Z_{\mathrm{I}}^{\prime}\right)+\left(-y \operatorname{Re} Z_{\mathrm{II}}^{\prime}\right), \\
& \hat{\sigma}_{x y}=\left(-y \operatorname{Re} Z_{\mathrm{I}}^{\prime}\right)+\left(\operatorname{Re} Z_{\mathrm{II}}-y \operatorname{Im} Z_{\mathrm{II}}^{\prime}\right) .
\end{aligned}
$$

In (7), we have assumed that the contact region is full bonded, so that the crack analogy can be used. This is equivalent to the infinite friction condition, or to the contact between two identical solids (regardless of the friction condition). For two different materials under finite frictional contact, the shear stress inside the contact should be considered 
in the calculation of image force. Finite friction usually does not change the load-displacement curve noticeably [18], but may change the stress distribution and thus lead to a large contribution to the image force.

The image force $J_{\text {image }}$ can be computed from the stress fields in (8) and the Peach-Koehler formula. The interaction force $J_{\text {int }}$ can be easily computed from (5) and (8) by substituting $-\mathbf{b}$ into $\mathbf{b}$, and 0 into $z_{0}$. The image force becomes less important when the contact size increases, since its magnitude decays rapidly with respect to the distance from the free surface. The interaction force decays with respect to the location of the leading dislocation.

We are concerned with the critical load or contact size to nucleate a dislocation. Using the Rice-Thomson criterion, a straightforward dimensional analysis gives that

$$
\frac{a_{\mathrm{crt}}}{b}=\Pi_{a}\left(\frac{\eta}{b}, \frac{\tau_{p}}{\mu}(1-\nu), \alpha, \theta\right) .
$$

After the dislocation is nucleated, the equilibrium position of the dislocation is determined by $J=\tau_{p} b$, leading to

$$
\frac{r_{\mathrm{eq}}}{a}=\Pi_{r}\left(\frac{a}{b}, \frac{\eta}{b}, \frac{\tau_{p}}{\mu}(1-\nu), \alpha, \theta\right),
$$

with $a>a_{\text {crt }}$. It is anticipated that when $a \gg b$, the driving force is dominated by the applied stress field, so that $r_{\mathrm{eq}} / a$ becomes size-independent since the applied stress field is selfsimilar. The relationship between $r_{\mathrm{eq}} / a$ and $a / b$ gives a qualitative measure of the dislocation density under the indenter.

When there are many dislocations, the driving force on the $k$ th dislocation consists of (1) and the interaction force from other pair of dislocations. The latter can be easily evaluated from (5) and (8). To determine the equilibrium positions of $N$ dislocations, we use the Newton-Raphson method to solve $N$ force-balance equations. The root finding process is very slow because of the $N$-dimensional functions and the shallow slope of driving force curves near equilibria.

\section{RESULTS AND DISCUSSION}

Figure 2 shows the total driving force with respect to the dislocation location. The zone where the interaction force dominates does not proportionally increase with the contact size. Consequently, the driving force on a fictitious dislocation at $r=\eta$ will eventually be nonzero and larger than the Peierls stress, assuming that the Peierls stress is smaller than $J_{\text {appl }}(r=0)$.

Figure 3(a) shows the critical contact size to nucleate one and many dislocations. The Rice-Thomson model introduces a length scale $\eta$, so that the first dislocation nucleation occurs at a finite contact size for this otherwise self-similar contact problem. It is found that the larger the wedge half-angle, the more difficult to nucleate a dislocation. The image force and interaction force are independent of the wedge angle. The applied driving force, however, is proportional to $\cot \alpha$. In addition, the shear stress near the wedge tip is bounded, so that there is a critical angle above which the maximum applied driving force is lower than the Peierls stress. The second and subsequent dislocations occur

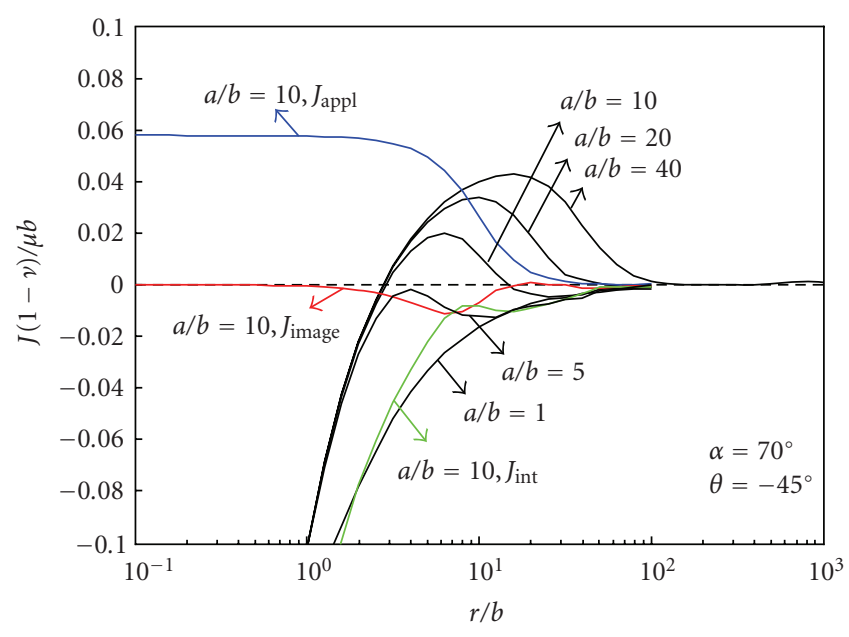

FIgURE 2: The configurational force on the dislocation plotted against the dislocation position with varying contact size.

at higher contact size, since the existing dislocation exerts strong back stress to prevent further dislocation nucleation. The diverging trend with respect to $\alpha$ is more evident, suggesting that dislocation multiplication for blunt wedges is extremely difficult. Consequently, when we are concerned with the effective dislocation number and density under a wedge contact, we must incorporate the bulk dislocation nucleation sources. The analysis presented in this paper is therefore only applicable for nanocontact experiments. This indenter geometry effects were also observed in a series of experiments $[6,19]$ where the sharper cube corner indenter tip (with half angle of $\sim 35^{\circ}$ ) shows higher hardness value as compared to the Berkovich indenter tip (with half angle of $\sim 70^{\circ}$ ) at the same indentation depth in the nanometer length scale, resulting in more dramatic indentation size effects.

Figure 3(b) shows the normalized dislocation equilibrium positions, $r_{\mathrm{eq}} / a$, with respect to the contact size. When $a / b \rightarrow \infty$, (10) will be independent of the contact size, and the effective plasticity zone will be proportional to the contact size. Consequently, the dislocation density is approximately linear with $b / a$ at large contact size. (Of course, when the contact size is large, it is the bulk nucleation sources and the statistically stored dislocations that govern the plastic zone and contact behavior.) However, this relationship will break down for small-contact size, because of the discrete nature of the dislocation nucleation and motion, and the length scale $\eta$. It thus becomes inappropriate to estimate plastic zone size in terms of contact size and relate the dislocation density to hardness in the nanohardness measurements. A more rational treatment would require a closer look at the connection between the mean contact pressure and the dislocation microstructure evolution at nanometer length scale.

\section{SUMMARY}

A dislocation mechanics study is performed here to examine the conditions for dislocation nucleation from, and 


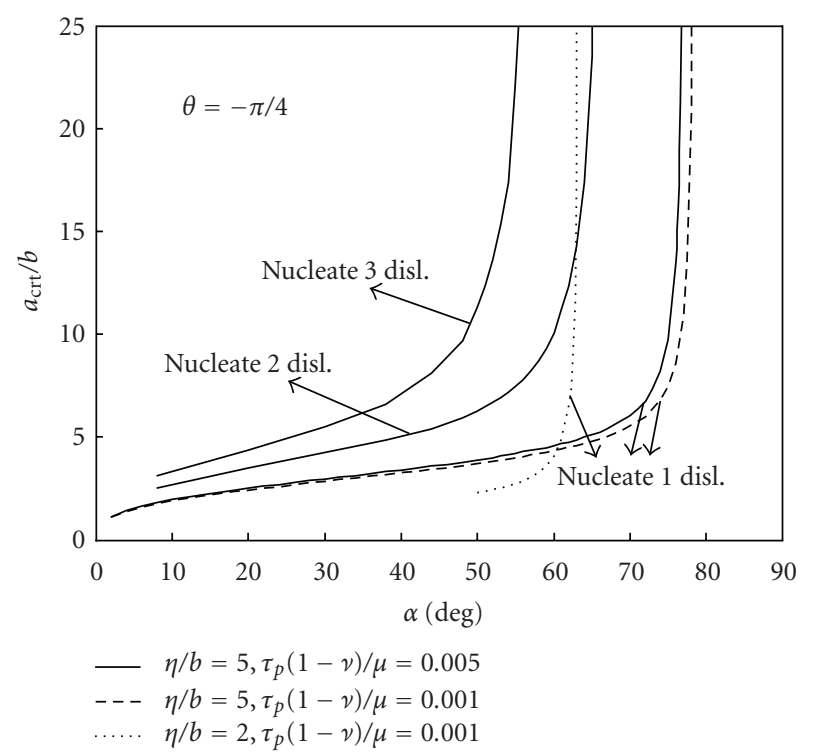

(a)

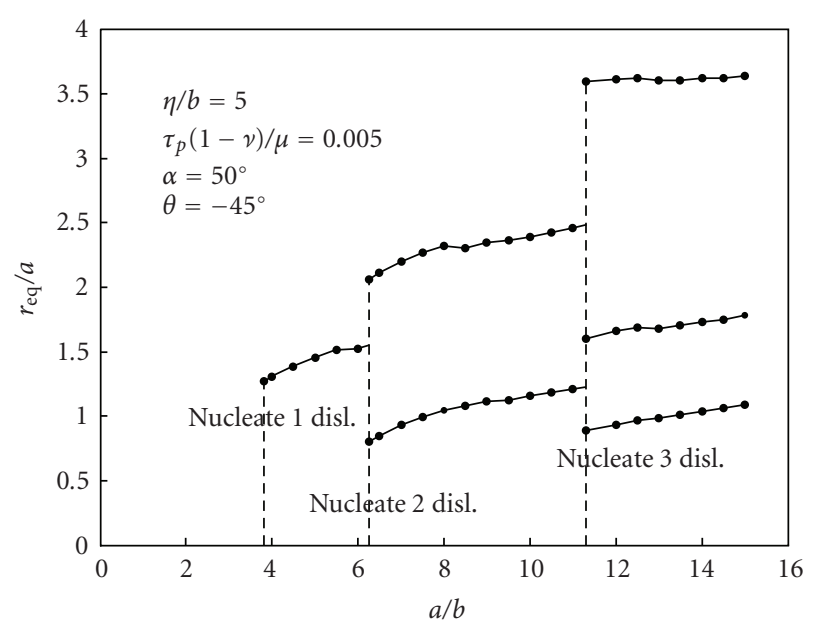

(b)

FIgURE 3: (a) The critical contact size to nucleate dislocations with respect to the wedge half-angle. (b) The equilibrium positions of nucleated dislocations with respect to the contact size.

pileup under, a wedge indenter. The configurational force on the dislocation is evaluated by linear elastic analysis, and the Rice-Thomson criterion is used for the dislocation nucleation. The contact strength map in Figure 3(a) shows that dislocation nucleation and multiplication are extremely difficult for blunt indenters, mainly because the stress state near the wedge tip consists of a finite shear stress and an infinite hydrostatic component. The ratio of the effective plastic zone size to the contact size is found to approach a constant when there are sufficient numbers of dislocations at large contact size. However, at nanoscale we should directly investigate the relationship between contact pressure and dislocation microstructure, instead of using dislocation-density-based hardening law.

\section{ACKNOWLEDGMENTS}

Y. F. Gao would like to acknowledge the support from the Joint Institute of Advanced Materials at the University of Tennessee. J. Lou gratefully acknowledges the startup fund from Rice University.

\section{REFERENCES}

[1] W. D. Nix and H. Gao, "Indentation size effects in crystalline materials: a law for strain gradient plasticity," Journal of the Mechanics and Physics of Solids, vol. 46, no. 3, pp. 411-425, 1998.

[2] J. G. Swadener, E. P. George, and G. M. Pharr, "The correlation of the indentation size effect measured with indenters of various shapes," Journal of the Mechanics and Physics of Solids, vol. 50, no. 4, pp. 681-694, 2002.

[3] J. Lou, P. Shrotriya, T. Buchheit, D. Yang, and W. O. Soboyejo, "Nanoindentation study of plasticity length scale effects in LIGA Ni microelectromechanical systems structures," Journal of Materials Research, vol. 18, no. 3, pp. 719-728, 2003.

[4] G. Feng and W. D. Nix, "Indentation size effect in MgO," Scripta Materialia, vol. 51, no. 6, pp. 599-603, 2004.

[5] Z. Zong, J. Lou, O. O. Adewoye, A. A. Elmustafa, F. Hammad, and W. O. Soboyejo, "Indentation size effects in the nano- and micro-hardness of fcc single crystal metals," Materials Science and Engineering A, vol. 434, no. 1-2, pp. 178-187, 2006.

[6] J. Lou, P. Shrotriya, S. Allameh, T. Buchheit, and W. O. Soboyejo, "Strain gradient plasticity length scale parameters for LIGA Ni MEMs thin films," Materials Science and Engineering A, vol. 441, no. 1-2, pp. 299-307, 2006.

[7] W. D. Nix, J. R. Greer, G. Feng, and E. T. Lilleodden, "Deformation at the nanometer and micrometer length scales: effects of strain gradients and dislocation starvation," Thin Solid Films, vol. 515, no. 6, pp. 3152-3157, 2007.

[8] H. Bei, E. P. George, J. L. Hay, and G. M. Pharr, "Influence of indenter tip geometry on elastic deformation during nanoindentation," Physical Review Letters, vol. 95, no. 4, Article ID 045501, 1-4, 2005.

[9] A. Widjaja, A. Needleman, and E. van der Giessen, "The effect of indenter shape on sub-micron indentation according to discrete dislocation plasticity," Modelling and Simulation in Materials Science and Engineering, vol. 15, no. 1, pp. S121-S131, 2007.

[10] Y. Huang, F. Zhang, K. C. Hwang, W. D. Nix, G. M. Pharr, and G. Feng, "A model of size effects in nano-indentation," Journal of the Mechanics and Physics of Solids, vol. 54, no. 8, pp. 16681686, 2006.

[11] K. L. Johnson, Contact Mechanics, Cambridge University Press, Cambridge, UK, 1985.

[12] J. R. Rice and R. Thomson, "Ductile versus brittle behavior of crystals," Philosophical Magazine, vol. 29, no. 1, pp. 73-97, 1973.

[13] H. H. Yu, P. Shrotriya, Y. F. Gao, and K.-S. Kim, "Microplasticity of surface steps under adhesive contact: part Isurface yielding controlled by single-dislocation nucleation," Journal of the Mechanics and Physics of Solids, vol. 55, no. 3, pp. 489-516, 2007.

[14] Y. F. Gao, B. N. Lucas, J. C. Hay, W. C. Oliver, and G. M. Pharr, "Nanoscale incipient asperity sliding and interface micro-slip assessed by the measurement of tangential contact stiffness," Scripta Materialia, vol. 55, no. 7, pp. 653-656, 2006. 
[15] Y. F. Gao, H. T. Xu, W. C. Oliver, and G. M. Pharr, "A comparison of Coulomb friction and friction stress models based on multidimensional nanocontact experiments," to appear in Journal of Applied Mechanics.

[16] Z. Suo, "Singularities interacting with interfaces and cracks," International Journal of Solids and Structures, vol. 25, no. 10, pp. 1133-1142, 1989.

[17] H. Tada, P. C. Paris, and G. R. Irwin, The Stress Analysis of Cracks Handbook, ASME Press, New York, NY, USA, 2000.

[18] Y. F. Gao, H. T. Xu, W. C. Oliver, and G. M. Pharr, "Effective elastic modulus of film-on-substrate systems under normal and tangential contact," Journal of the Mechanics and Physics of Solids, vol. 56, no. 2, pp. 402-416, 2008.

[19] R. A. Mirshams and P. Parakala, "Nanoindentation of nanocrystalline $\mathrm{Ni}$ with geometrically different indenters," Materials Science and Engineering A, vol. 372, no. 1-2, pp. 252260, 2004. 

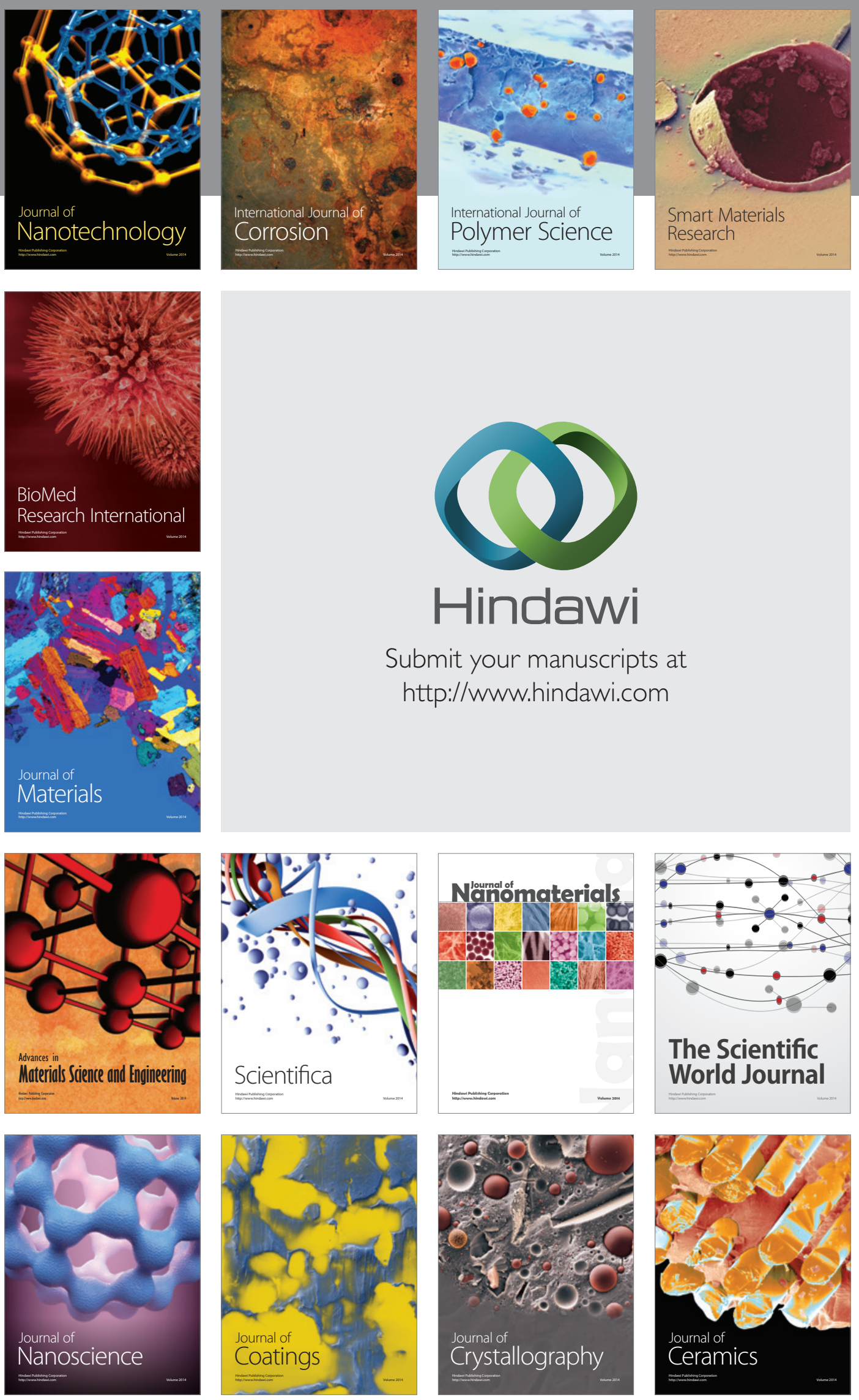

The Scientific World Journal

Submit your manuscripts at

http://www.hindawi.com

\section{World Journal}

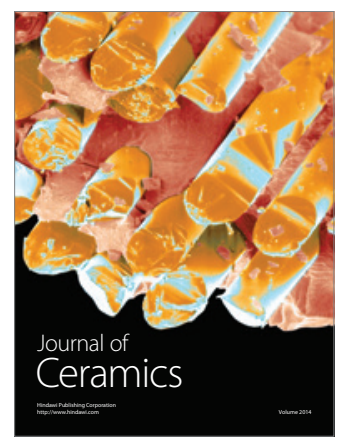

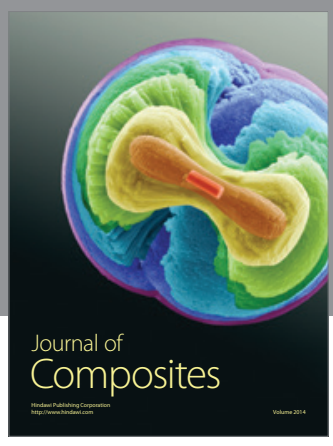
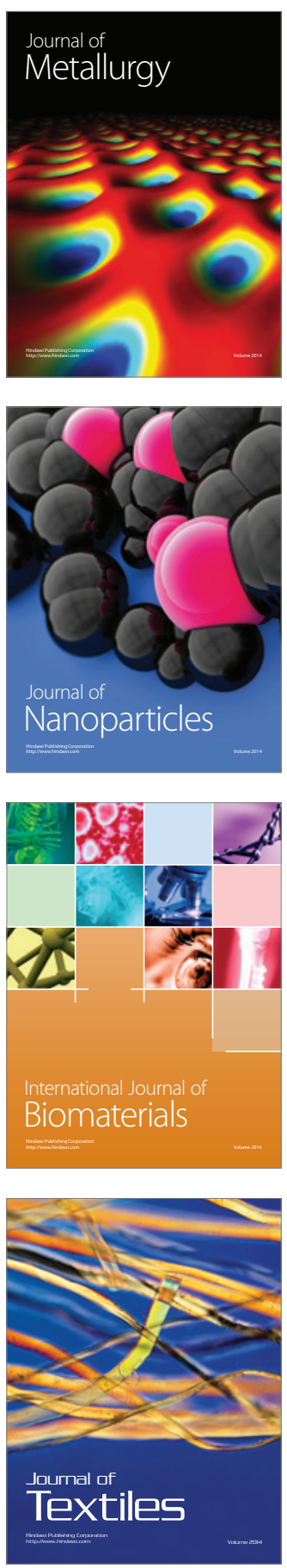